\title{
Effect of hand tactile stimulation on the vital signs of brain injury patients hospitalized in Qazvin intensive care units, Iran
}

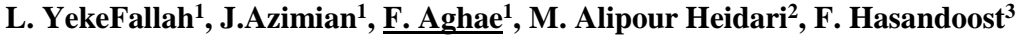

\author{
${ }^{1}$ Department of Critical Care Nursing, School of Nursing and Midwifery, Qazvin University of Medical Sciences, Qazvin, \\ Iran \\ ${ }^{2}$ Department of Statistics, Qazvin University of Medical Sciences, Qazvin, Iran \\ ${ }^{3}$ Tarbiat Modares University, Tehran, Iran \\ Corresponding Address: Fatemeh Aghae, Department of Critical Care Nursing, School of Nursing and Midwifery, Qazvin \\ University of Medical Sciences, Qazvin, Iran \\ Tel: +98-919-1804286, Email: faghae17711@gmail.com \\ Received: 8 Apr 2018; Accepted: 4 Sep 2018
}

* Abstract

Background: Hospitalization in intensive care units (ICUs) results tactile deprivation, which may cause physiologic imbalance.

Objective: This study aimed to investigate the effect of hand tactile stimulation on the vital signs of traumatic head injury patients in Qazvin ICUs.

Methods: This clinical trial study was conducted on sixty patients with severe brain trauma admitted to ICUs in Qazvin, in 2016. The subjects were selected by the convenience sampling method and assigned in two groups of intervention and control. Hands, wrists and palms of patients were touched for 5 minutes twice a day (morning and evening) and vital signs were recorded immediately from the 1 st to 5 th day of admission. Data were analyzed by the paired and independent $t$ tests.

Findings: According to the results, hand tactile stimulation significantly decreased systolic blood pressure level $(\mathrm{P}=0.0001)$, diastolic blood pressure $(\mathrm{P}<0.05)$ and respiration rate $(\mathrm{P}<0.05)$ in the intervention group. However, tactile stimulation had no significant effect on the body temperature of the patients $(\mathrm{P}>0.05)$.

Conclusion: As the positive effect of hand tactile stimulation on decrease of heartbeat rate and blood pressure in patients with brain trauma, it is recommended to use tactile stimulation methods on ICUs patients.

Keywords: Touch, Vital signs, Traumatic brain injuries, Sensory deprivation, Intensive care units Citation: Effect of hand tactile stimulation on the vital signs of brain injury patients hospitalized in Qazvin intensive care units, Iran. J Qazvin Univ Med Sci 2018; 22(4): 13-21. 


\title{
تأثير لمس دست بر علايم حياتى بيماران ضربه مغزى بسترى در بخش ICU مراكز درمانى شهر قزوين
}

\author{
دكتر ليلى يكه فلاح'، دكتر جليل عظيميان'، فاطمه آقائي'، دكتر محمود عليبور حيدرى '، فاطمه حسن دوستَ
}

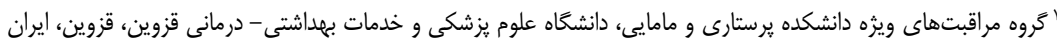

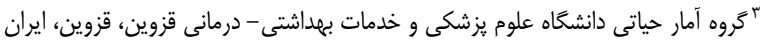

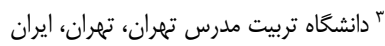

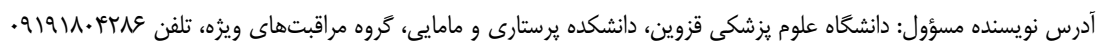
تاريخ دريافت:

زمينه: بسترى در بخشهاى مراقبت ويزه منجر به محروميت حسى شده و مى تواند تعادل فيزيولوزيكى بيمانيمار را مختل كند.

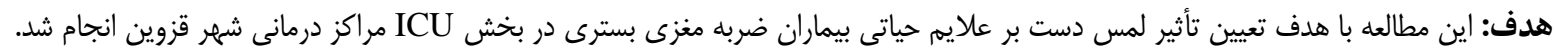

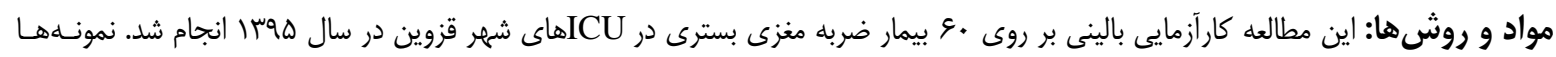

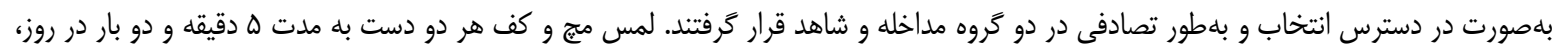

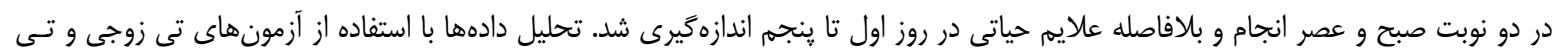
مستقل انجام شد.

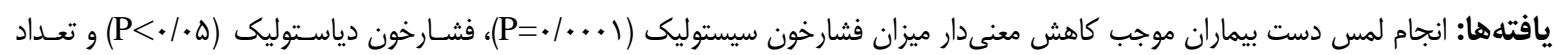

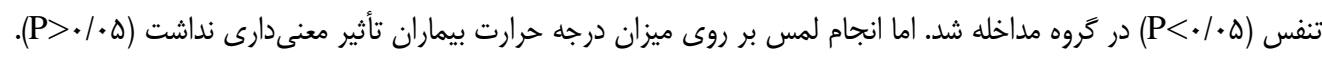

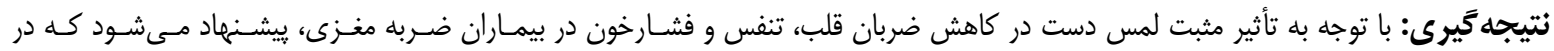
بخشهاى ويثه تحريك لمسى به كار كرفته شود.

كليدوازهها: لمس، علايم حياتى، ضربه مغزى، محروميت حسى، بخش مراقبتهاى ويزه

از بيمار، بيشتر استرس ايجاد مى كند.ه(-1) قرار خـرفتن در

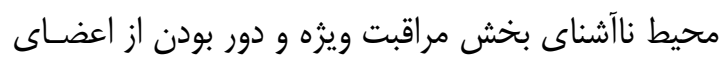

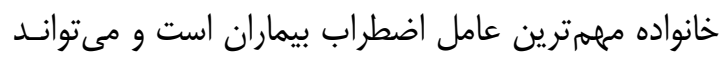

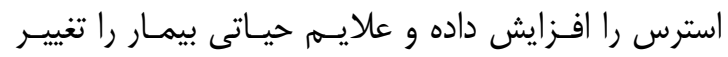

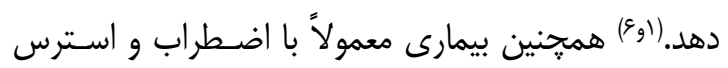

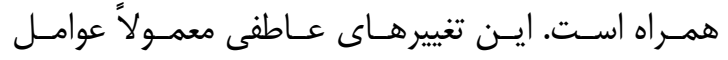

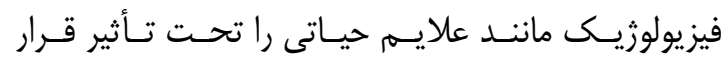

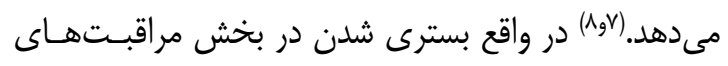

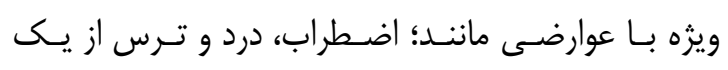

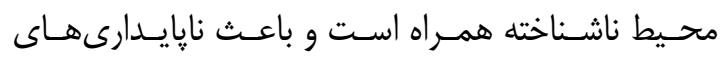

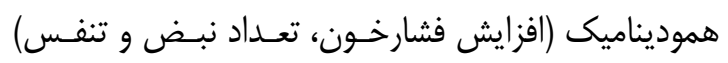

مقدمه:

بيماران ترومايى بسترى در بخش مراقبتهـاى ويـرّه

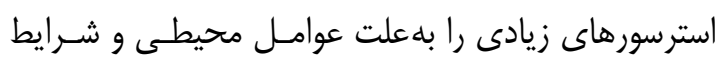

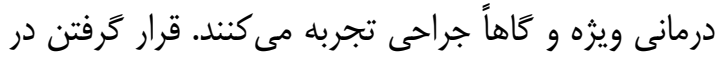

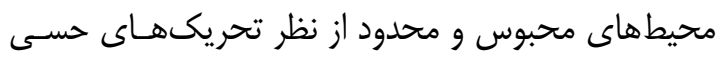

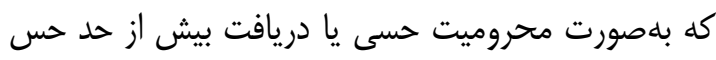

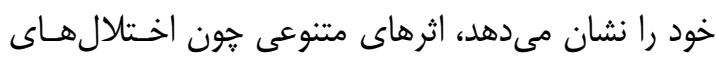

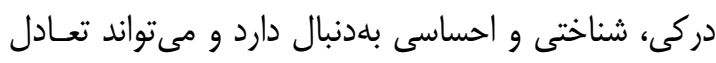

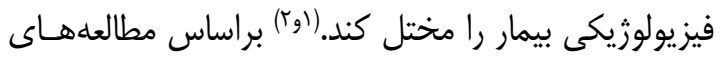

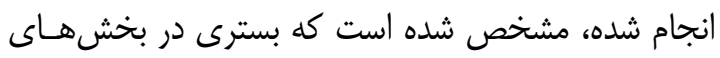

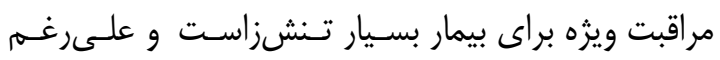

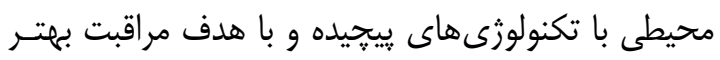


اقتصادى، غيرتهاجمى و غيردارويسى بـوده و كامـل كنتـده

مراقبت يزشكى است. (1ه)

لمس بخش ذاتى از مداخلههاى يرستارى است كه به اسه

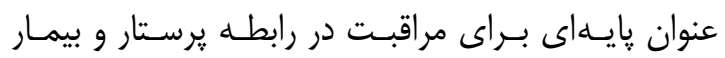

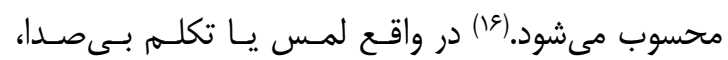

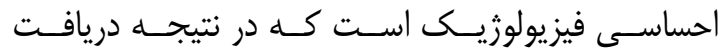

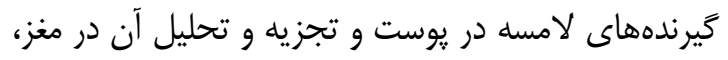

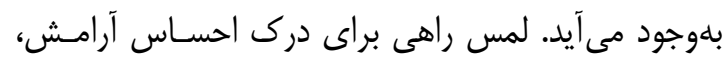

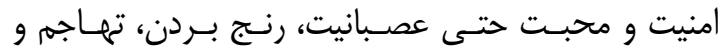

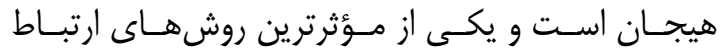

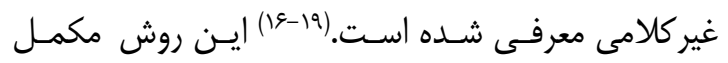

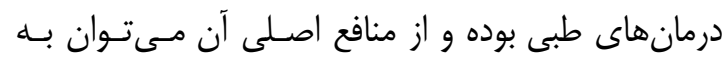

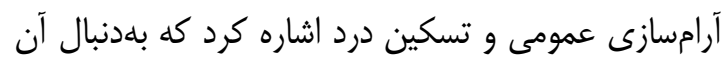

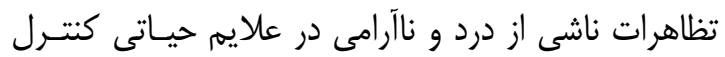

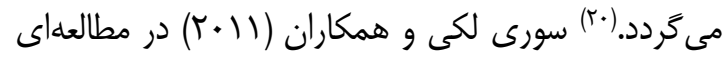
با عنوان اثر لمس بر روى اكسيثن خون شريانى بيمـاران

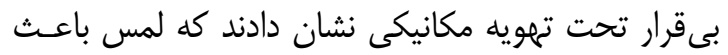

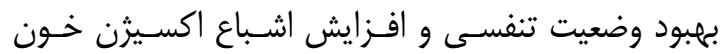

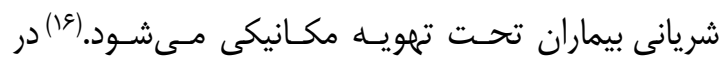

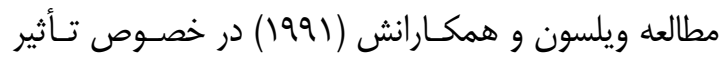

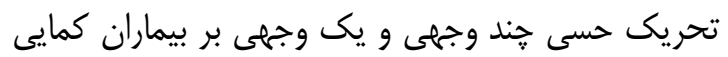

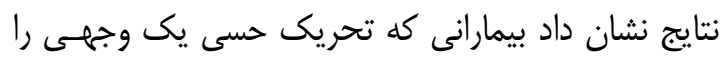

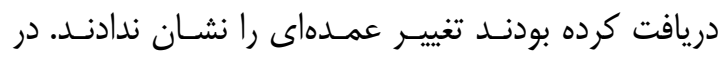

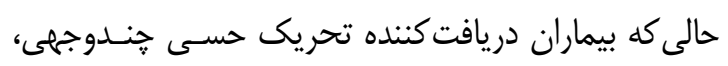

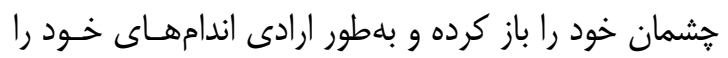

$$
\text { حركت مى حدادند.) حتان }
$$

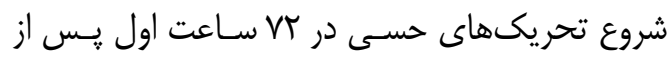

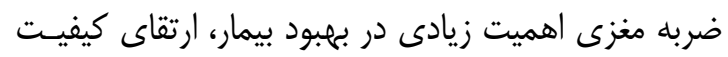

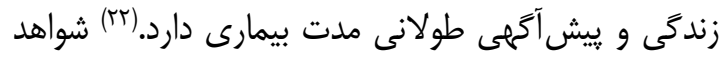

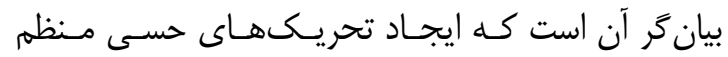

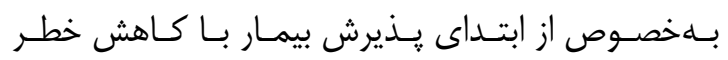

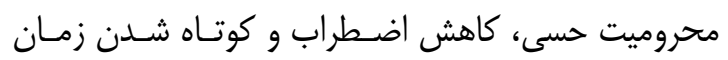

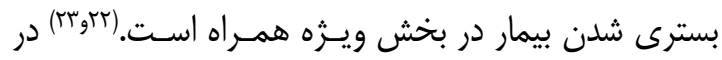

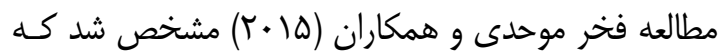

مى گردد.

سالانه حدود //ه ميليون مـرى در نتيجـهـ آسـيبهـا

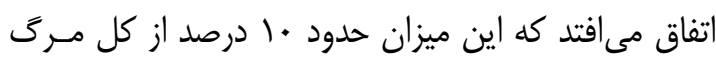

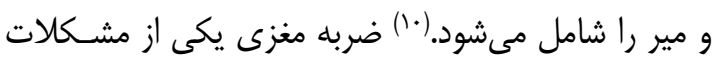
عمده در سراسر جهان و از مهمترين عوامل مركى و ميـر

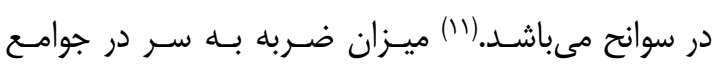

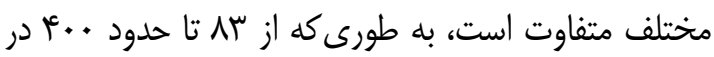

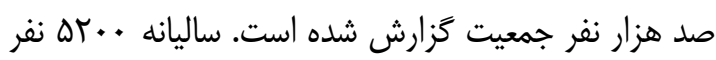

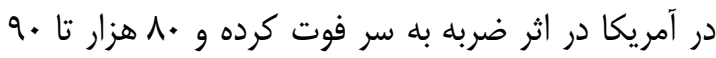

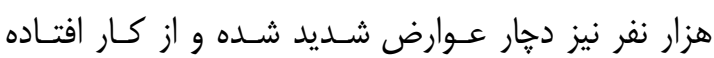

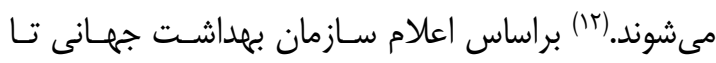

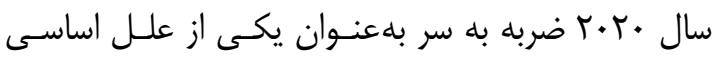

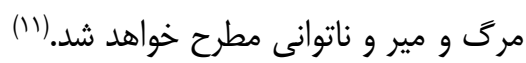

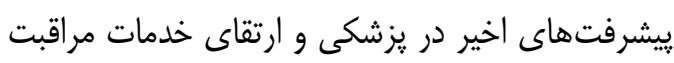

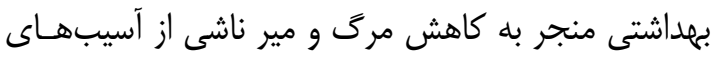

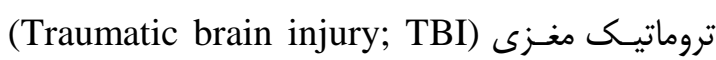
شده است. هر جند اين يِيشرفتها مى توانند منجر به حفظ

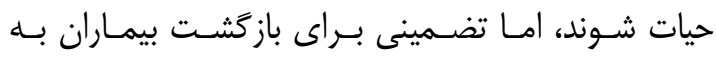

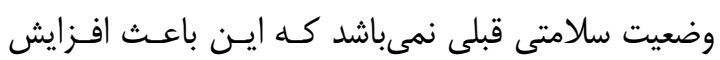

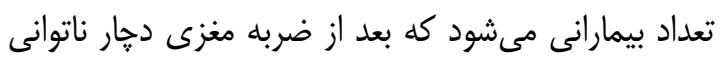

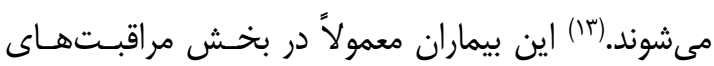

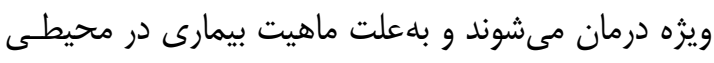

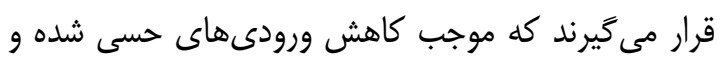

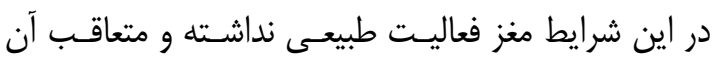
دجار محروميت حسى مىشود.

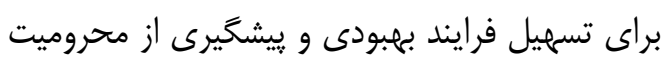

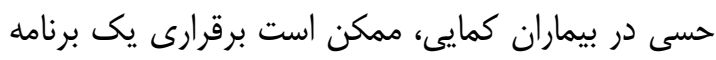

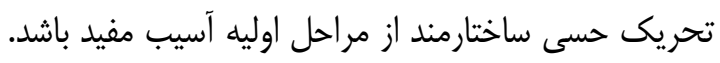

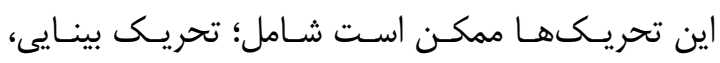

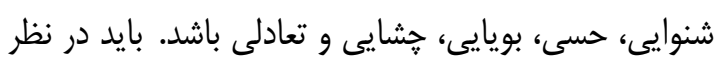

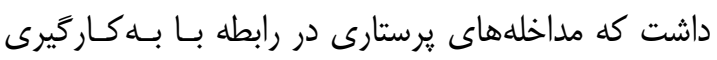

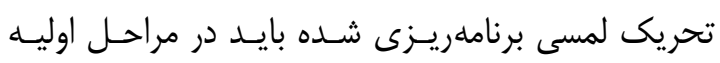

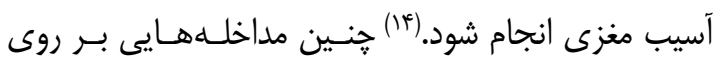
تمـام بـدن شخـص (ذهن، جسم و روح) تأثير كذاشتـهـ و 
•r بيمار بهصورت تصادفى در گروه مداخله و •r بيمار در

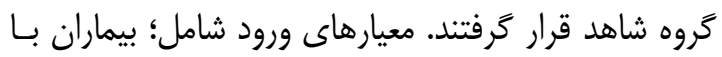

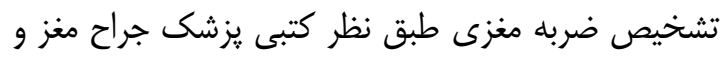

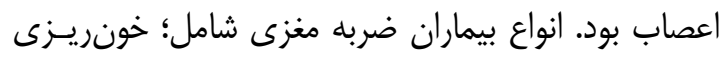

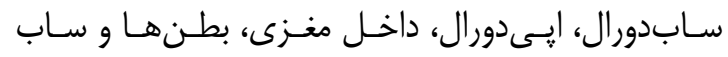

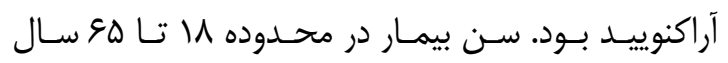

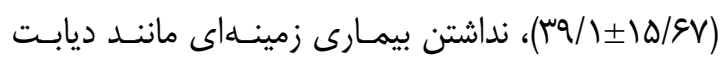

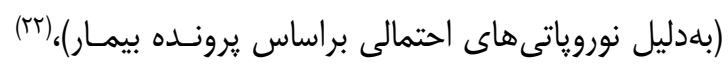

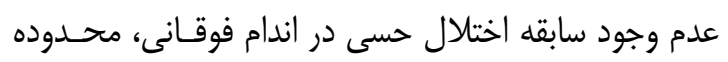

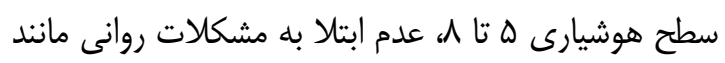

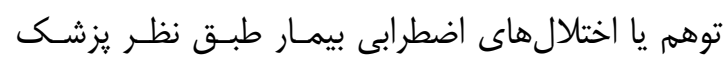

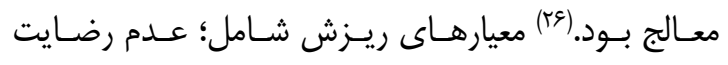

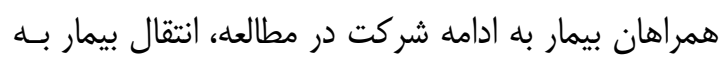

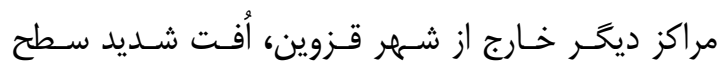

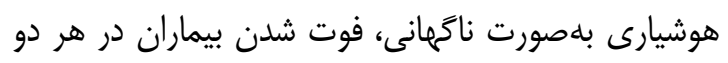

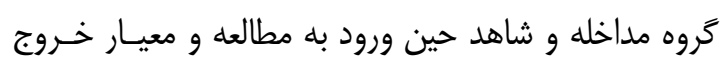
شامل؛ شركت همزمان بيمار در برنامههاى مراقبتى مشابه مدابه

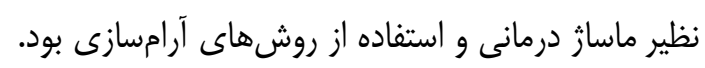
روش اجراى كار بهاين صورت بود كه گَروه مداخلـه، تحت لمس مج دست و كف هر دو دست بيمار از مـج بـــ

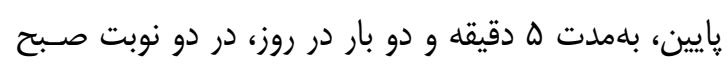

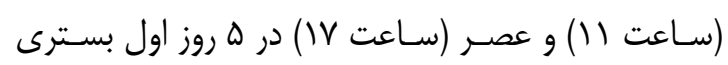

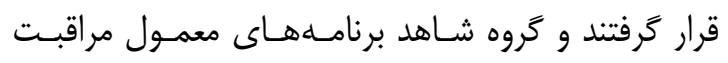

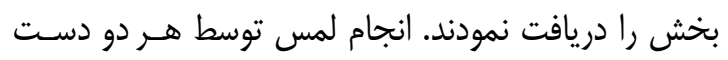

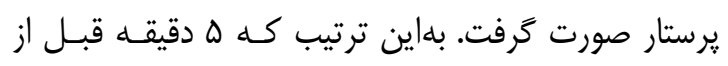

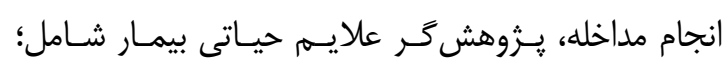

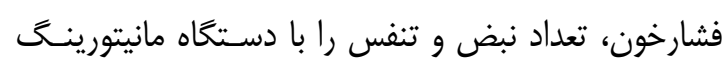

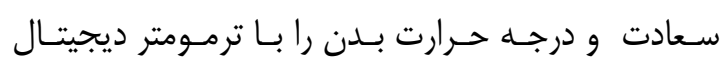

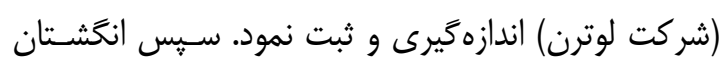

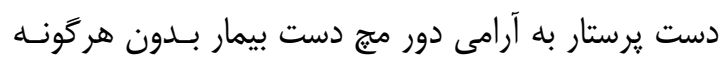
اعمال فشارى قرار و يس از آن كل دست از مج به دئ يـايين

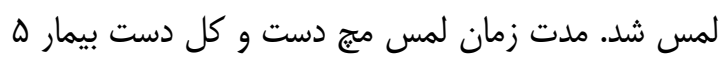

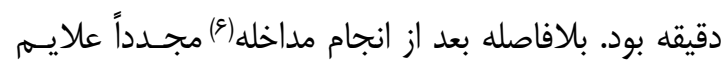

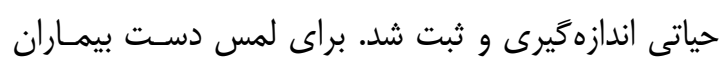

لمس در كاهش علايم حياتى بيماران نقش دارد. با توجـهـ

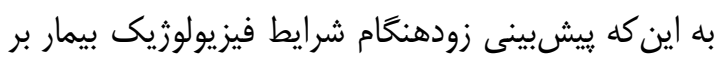

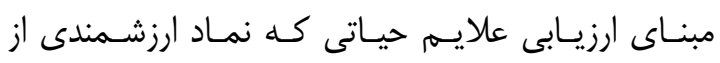

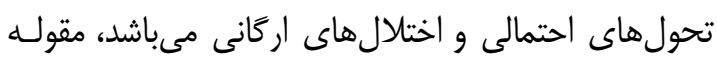

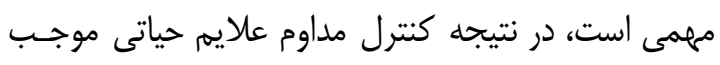

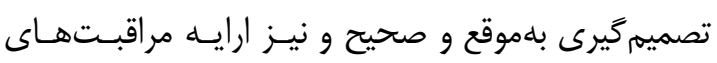

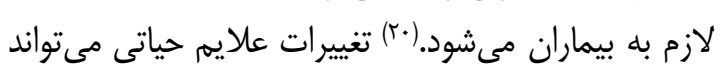

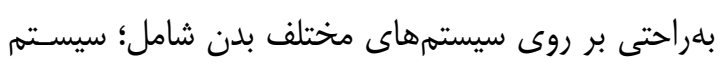

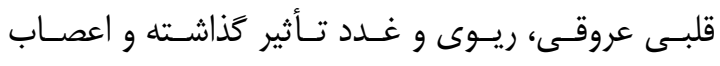

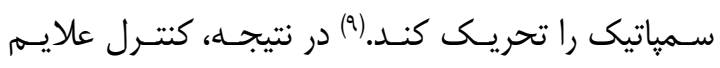

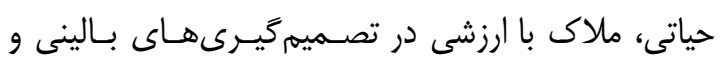

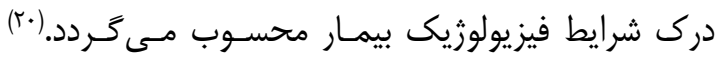

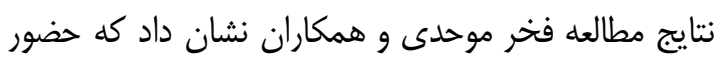

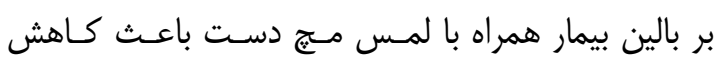

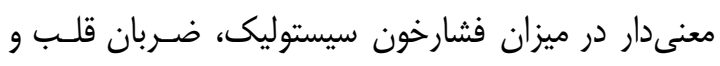

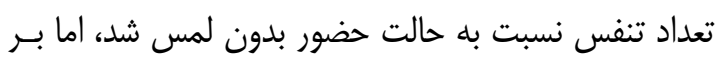

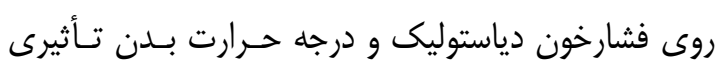

نداشت. (r.)

تحريكهاى حسى باعث تحريك شبكه عصبى شـده

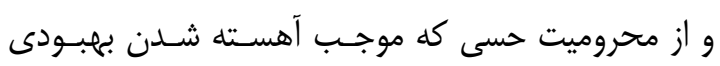

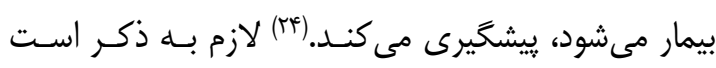

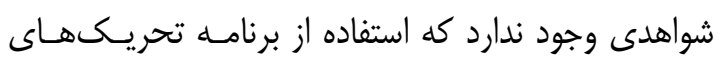

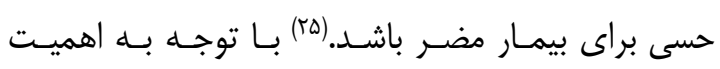
مداخله هاى غيردارويسى يرسـتارى، در ايـن مطالهـه تـأثير

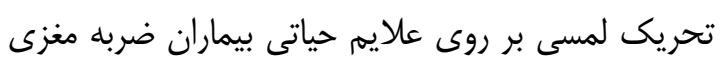
در بخشهاى مراقبت ويزه تروماى مراكز تأمين اجتماعى دئى

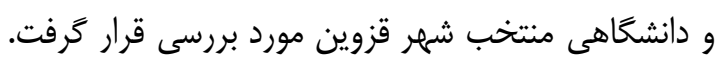

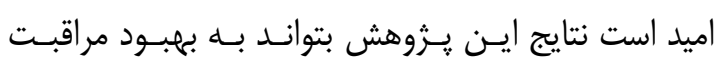
يرستارى و كاهش زمان بسترى بيماران كمك نمايد.

\section{مواد و روشها:}

اين مطالعه يك طرح كارآزمايى بالينى است كه جامعه

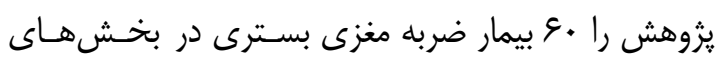

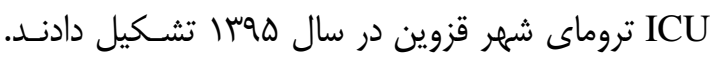


نبض بيماران در كروه مداخله، به ميزان /// عدد كـاهش

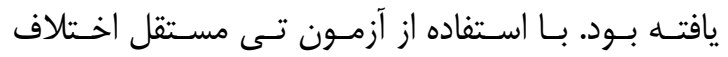

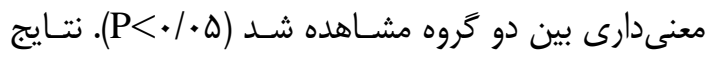

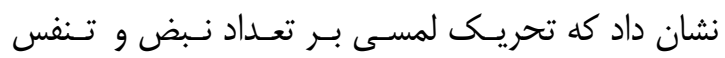

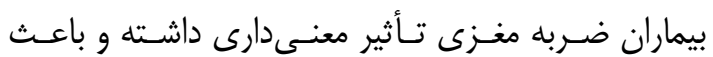
كاهش تعداد نبض و تنفس گرديد.

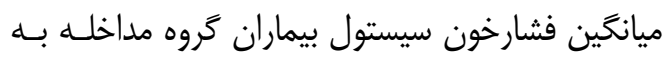

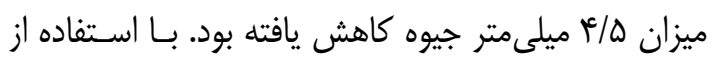

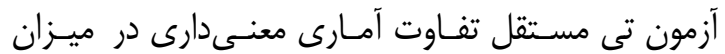

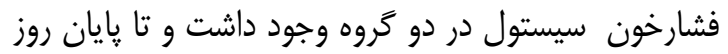

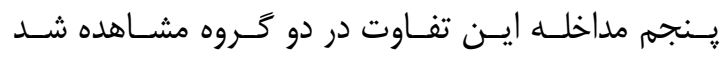
ماخله

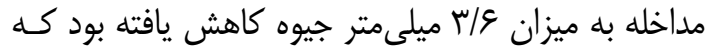

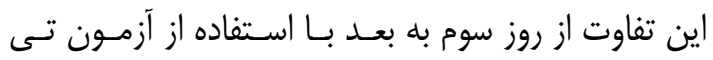

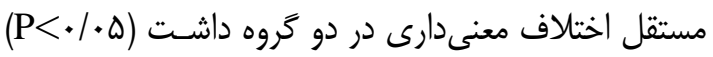

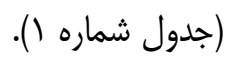

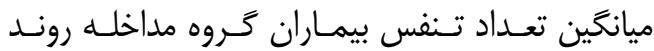

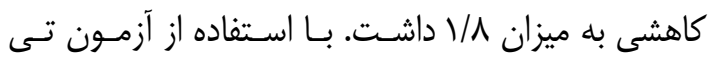

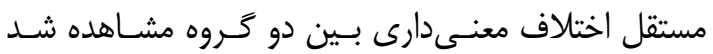

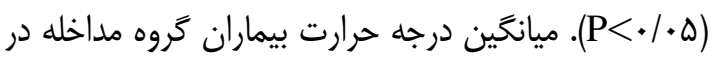

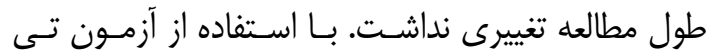

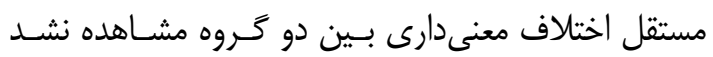

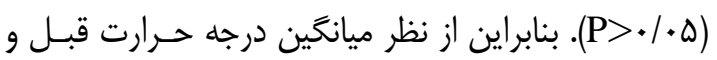

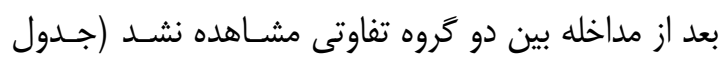

شماره r).
مؤنث توسط يزوهش كر و براى لمس دست بيماران مذكر

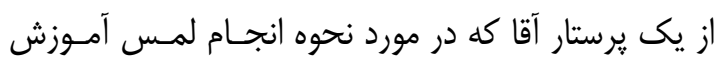
ديده بود در حضور يزوهش إنر استفاده شد. در اين يزوهش تخصيص نمونه ها در كروه مداخلـه و

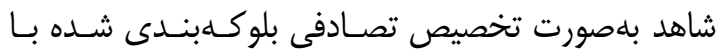

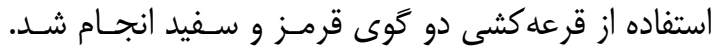

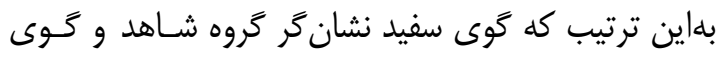

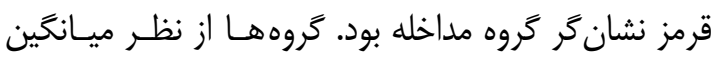
سطح هوشيارى روز اول همگن بودند. براى تحليل دادهها

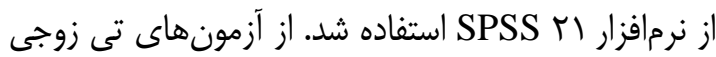

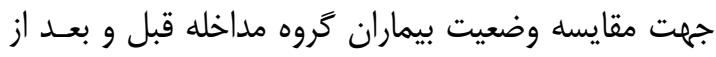

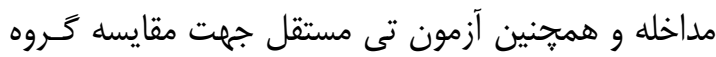
مداخله و شاهد استفاده شد. سـطح معنسى دارى كـمتــر از ملاز

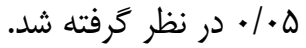

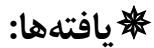

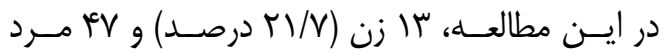

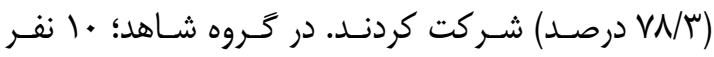

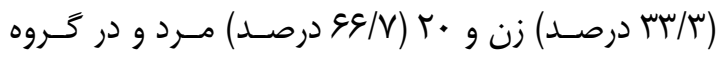

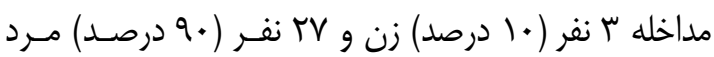

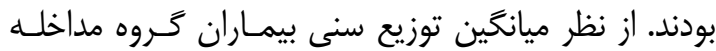
إ كروه شاهد با استفاده از آزمون تى مستقل تفاوت آمـارى معنسى دارى

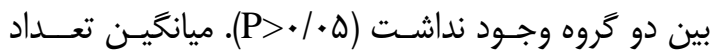

جدول ( - مقايسه ميانكَين علايم حياتى (نبض و فشارخون) در دو تروه مورد مطالعه

\begin{tabular}{|c|c|c|c|c|c|c|c|}
\hline \multicolumn{6}{|c|}{ انحراف معيار 土 ميانخين } & \multirow{2}{*}{\multicolumn{2}{|c|}{ علايم حياتى }} \\
\hline \multicolumn{2}{|c|}{ فشارخون دياستوليك } & \multicolumn{2}{|c|}{ فشارخون سيستوليك } & \multicolumn{2}{|c|}{ تعداد نبض } & & \\
\hline بعد از مداخله & قبل از مداخله & بعد از مداخله & قبل از مداخله & بعد از مداخله & قبل از مداخله & زمان انجام مداخله & (روز \\
\hline$V Q / \digamma \wedge+\mid f / F \wedge$ & $V \varepsilon / T r+19 / V V$ & $I r F / V H+r$. & $1 \pi \cdot \pi+r / r q$ & AT/TH+Ir/Mr & $\Lambda T / V \Delta+I r / \Gamma \Lambda$ & آزمون & \multirow{2}{*}{ 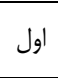 } \\
\hline$V T / K r+I N / S$ & $V E / T V+I V / T \Lambda$ & $I r \cdot / V \Delta+r r / \cdot q$ & $1 r \cdot / 9 r+r r / g r$ & $\Lambda T / T H+I T / K r$ & $\Lambda r / V \Delta+I r / F \Lambda$ & شاهد & \\
\hline$V T / G+I T / F T$ & $V \Delta / \Delta r+1 f / \Delta q$ & $11 / \wedge \Delta+19 / 14$ & $|r \varepsilon / \Delta+r| / V$. & NI/AI+IT/Kr & $\Lambda T / \cdot V+1 Y / r$ & آزمون & \multirow[b]{2}{*}{ 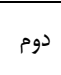 } \\
\hline$V \leftarrow / \cdot \Delta+I V / \Lambda \Delta$ & $V V^{c} / \varepsilon+I V / \wedge r$ & $\mid r \cdot / I \varepsilon+Y Y / \cdot 9$ & $|r| / \Delta \varepsilon+r M / \& \Lambda$ & NI/NI+IT/TK & $\Lambda r / \cdot V+I r / r$ & شاهد & \\
\hline$V) / 9 V+11 / 9 f$ & $V \varepsilon / J+\mid Y / \cdot r$ & $|V V / q \Delta+r| / \cdot 9$ & $1 Y N / q \Delta+r+/ \Delta r$ & $\Lambda \cdot / \Lambda \Delta+\mid 1 / /)^{f}$ & $\Lambda 1 / \cdot \Lambda+I I / r f$ & آزمون & \multirow[b]{2}{*}{ سوم } \\
\hline$V F / \Delta V+I D / H Y$ & $V \varepsilon / Q \Lambda+1 Q / r^{2}$ & $\| r \cdot|\Delta D+r| / R^{c} r$ & $|r| / f+\mid N / q q$ & $\Lambda \cdot / \Lambda \Delta+\mid 1 / /)^{f}$ & $\Lambda 1 / \cdot \Lambda+11 / K^{c}$ & شاهد & \\
\hline$V T / \mu I+I T / \mu r$ & $V V / T r+I Q / F q$ & $I I V / V \wedge+I \Delta / \wedge V$ & $1 K E / T H+19 / 4 t$ & $\Lambda T / T+1 \cdot / T g$ & $11 / f+1+/ 4 f$ & آزمون & \multirow[b]{2}{*}{ ") } \\
\hline$V Q / Q \Psi+I Q / V \Lambda$ & $V Q / I V+I Q / Q Y$ & $|T r / 9 \Lambda+| N / 9 V$ & $|T T / T V+| N / F \Delta$ & $\Lambda T / T H+1 \cdot / K \varepsilon$ & $\wedge 1 / f+1 \cdot / 4 f^{c}$ & شاهد & \\
\hline$V T / r I+I T / q g$ & $V V^{c} / 91+10 / 9$ & $119 / \cdot \Delta+1 f / r$. & $|Y V / V \Delta+| N / I \mid$ & $V E / V G+q / \Delta T$ & Vq/VI+q/Ar & آزمون & \multirow{2}{*}{ ينجم } \\
\hline$V D / \cdot \varphi+1 f / \Delta \varphi$ & $V \Delta / \& I+\mid f / F \Delta$ & $I T T / T \omega+I N / \Lambda \Lambda$ & $1 \% T / 9 T+19 / T V$ & $V E / V G+q / \Delta T$ & Vq/VI+q/Ar & شاهد & \\
\hline
\end{tabular}




\section{جدول r - مقايسه ميانكَين علايم حياتى (تنفس و درجه حرارت) در دو تروه بيماران ضربه مغزى بسترى در بخش ICU}

\begin{tabular}{|c|c|c|c|c|c|}
\hline \multicolumn{4}{|c|}{ انحراف معياري ميانگين } & \multirow{2}{*}{\multicolumn{2}{|c|}{ علايم حياتى }} \\
\hline \multicolumn{2}{|c|}{ درجه حرارت } & \multicolumn{2}{|c|}{ تعداد تنفس } & & \\
\hline بعد از مداخله & قبل از مداخله & بعد از مداخله & قبل از مداخله & زمان انجام مداخله & روز \\
\hline$r V / \Delta T+\cdot / \Delta Q$ & $T V / \Delta Q+\cdot / \Delta T$ & $|Q / T|+r / q Y$ & $|\omega / q|+f / \& \Delta$ & آزمون & \multirow{2}{*}{ اول } \\
\hline$r V / \Delta \cdot+\cdot / q V$ & $r V / \Delta \mid+\cdot / \varepsilon \Delta$ & $1 \varepsilon / \mu \omega+\mu / \kappa$. & $18 / \mu r+r / 8 D$ & شاهد & \\
\hline$r V / \Delta \cdot+\cdot / \Delta \Delta$ & $\Gamma V / \Delta \Delta+\cdot / \mathcal{G}$ & $1 r / \cdot r+r / \wedge q$ & $\mid \varepsilon / \cdot r+r / \Lambda F$ & آزمون & \multirow[b]{2}{*}{ دوم } \\
\hline$r V / \Psi A+\cdot / G \psi$ & $r V / \& q+\cdot / q \mu$ & $1 \varepsilon / \Gamma+\Gamma / \kappa \Delta$ & $1 \xi / \Gamma+r / \Delta$. & شاهد & \\
\hline$r V / \Delta S+\cdot / \Delta T$ & $r V / \Delta \Lambda+\cdot / \Delta r$ & $1 \Psi / \Lambda \Upsilon^{2}+r / V$ & $1 \omega / I r+r / q r$ & آزمون & \multirow[b]{2}{*}{ 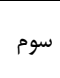 } \\
\hline$r V / 4 F+\cdot / \Delta q$ & $r V / \& \Delta+\cdot|q|$ & $|\varepsilon / \mu \Lambda+r / \Delta|^{F}$ & $\mid \varepsilon / \mathcal{L}+r / \Delta T$ & شاهد & \\
\hline$r v / c q+\cdot / c q$ & $r V / \Delta)+\cdot / q^{c} V$ & $1 r / 9 r+r / \Lambda$ & $\mid \omega / g r+r / \Lambda \Delta$ & آزمون & \multirow{2}{*}{ جههارم } \\
\hline$r V / \mathcal{F} \Delta+\cdot / \bar{q} \mu$ & $r V / \varphi \&+\cdot / \Delta \Lambda$ & $|\varepsilon / \mathcal{f}+r /| V$ & $\mid \varepsilon / \gamma \lambda+r / I V$ & شاهد & \\
\hline$r V / \Delta T+\cdot / \Delta T$ & $r V / F V+\cdot / F V$ & $I F / V \Delta+r / r \Lambda$ & $\mid \omega / \Delta D+r / F \psi$ & آزمون & \multirow{2}{*}{ ينجمم } \\
\hline$r V / \& \Delta+\cdot /<q$ & $r V / F \Delta+\cdot / \Delta$ & $|\varepsilon / \backslash \Lambda+\mu /| q$ & $1 \varepsilon / r+r / \mid r$ & شاهد & \\
\hline
\end{tabular}

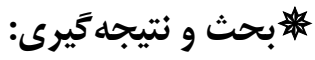

وجود داشت و لمس درمانى باعث كـاهش تعـداد ضـربان

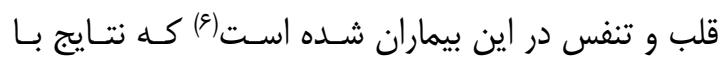
مطالعه ما همسو مىباشد.

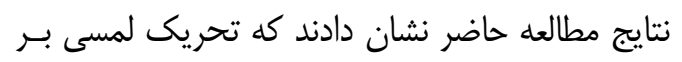

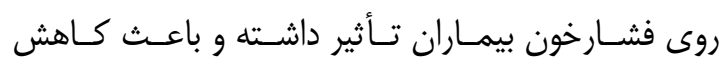

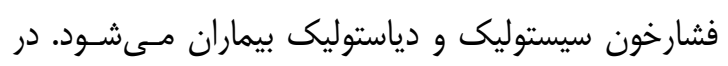

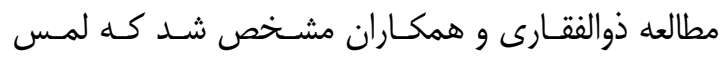

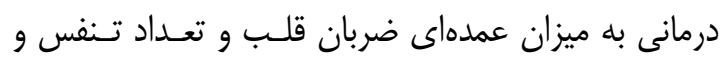

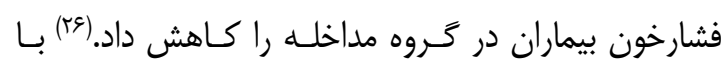

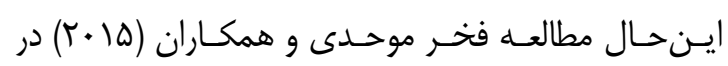

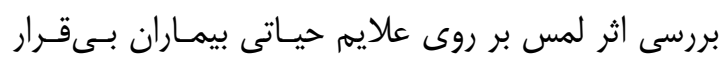

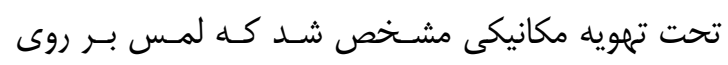

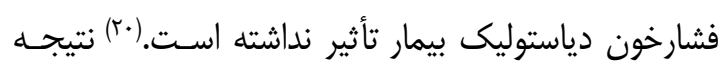

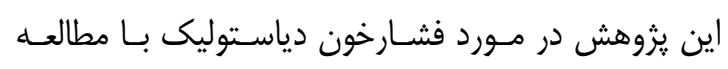

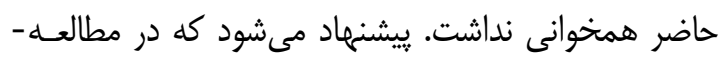

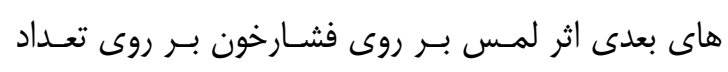
نمونهاى بيشترى انجام كيرد.

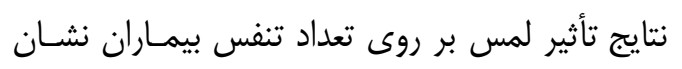

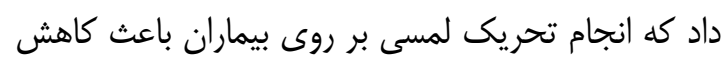
تعداد تنفس مى شود. در مطالعه سـورى لكى لـى و همكـاران

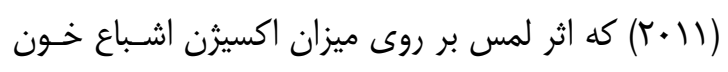

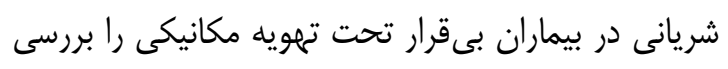

اين مطالعه نشان داد كه لمس دست بر تعديل عيرئ علايم

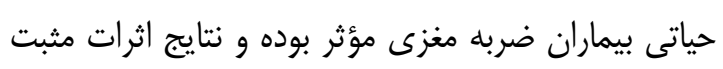

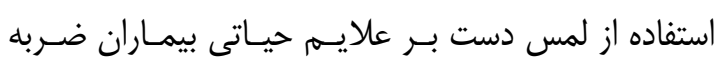

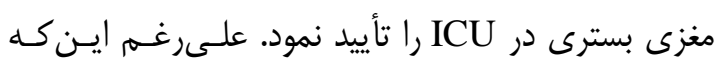

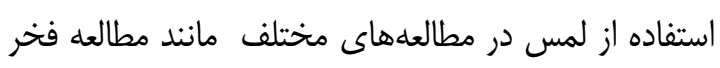

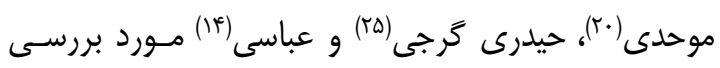

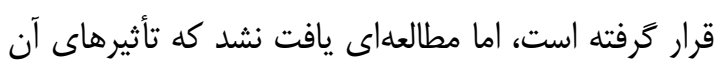
بر علايم حياتى بيماران كمايى را مورد بررسـى قـرار دادئه

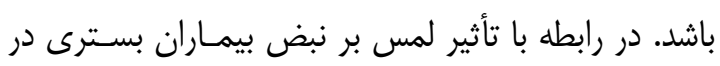

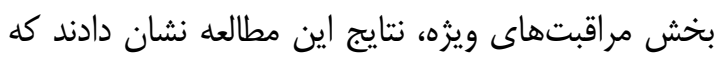

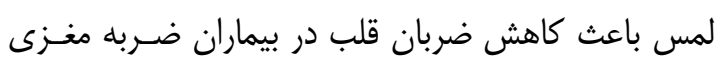

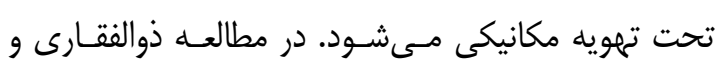

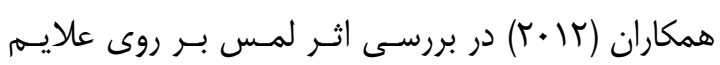

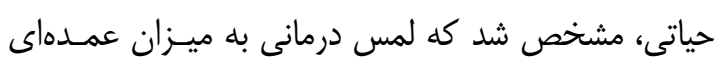

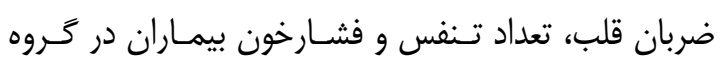

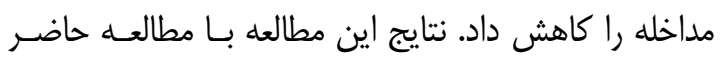

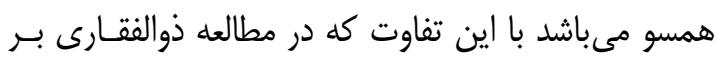

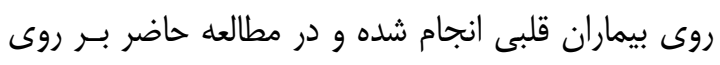

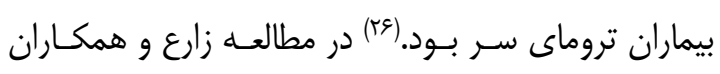

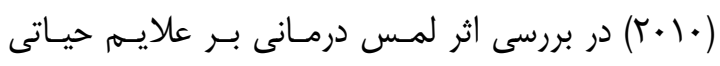

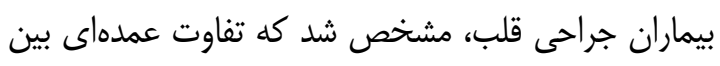

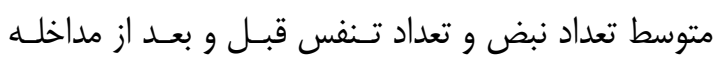


الهام بخش تحقيقهاى ديخر در اين زمينه و زمينـهـــاى

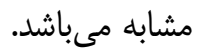

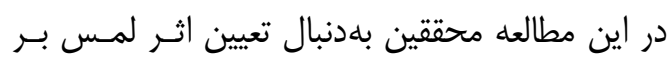

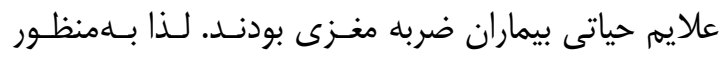
كنترل بهتر متغيرهاى مخدوش كننده احتمالى، اين مطالعه

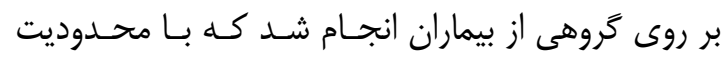

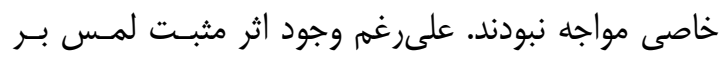

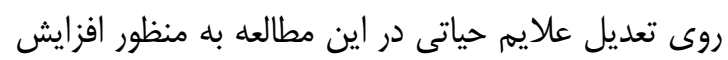

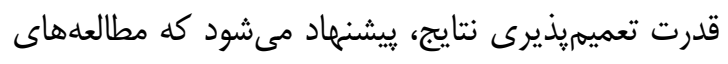

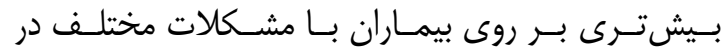

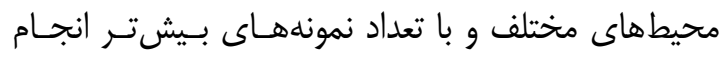

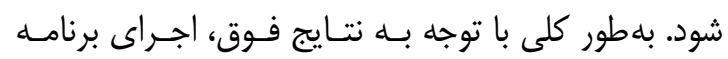
تحريك لمسى منظه بر روى علايم حياتى بيماران ضـربه

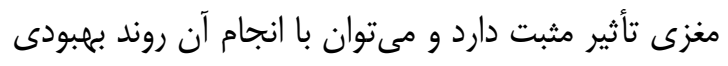

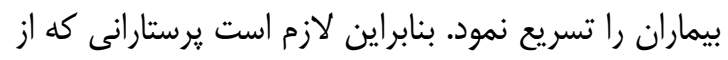

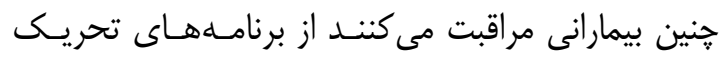

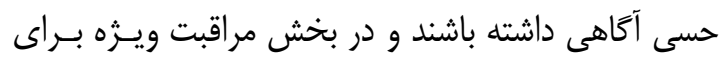

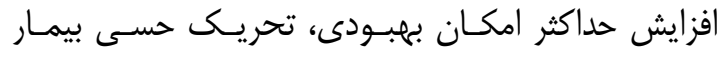

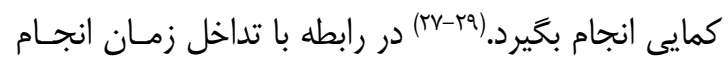

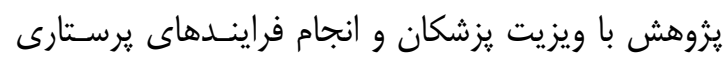

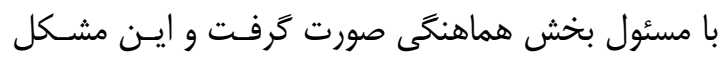

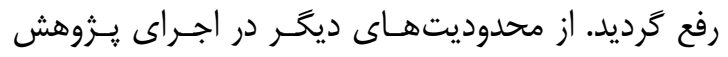

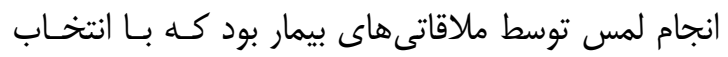

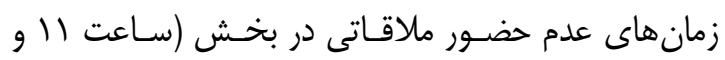
اين محدوديت كنترل گرديد.

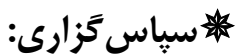

بدينوسيله از تمام بيماران و خانوادههاى آنان كـه در اجراى مطالعه مشاركت داشتند، تقدير و تشكر مسى تـرد دد.

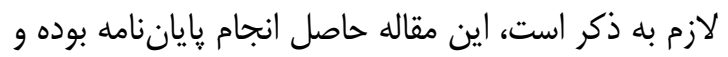

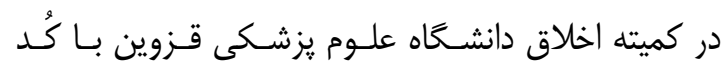

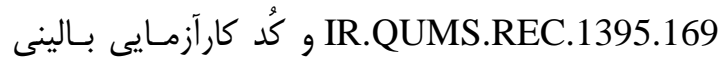
ثبت كرديد. IRCT2016062128563N1
كردند، مشخص شد كه لمس وضعيت تنفسى بيمـاران را

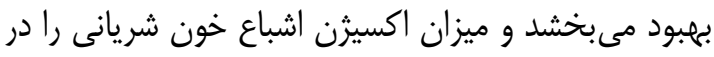

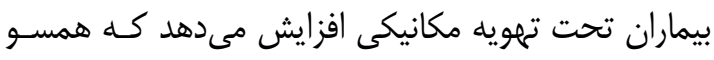

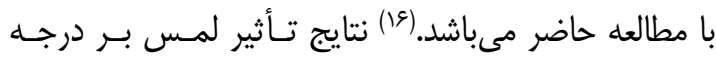
حرارت بيماران بسترى در بخش مراقبتهاى ويزه نشـان

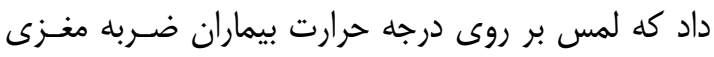

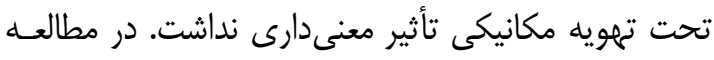

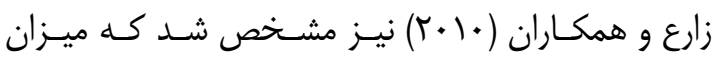

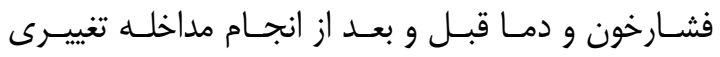

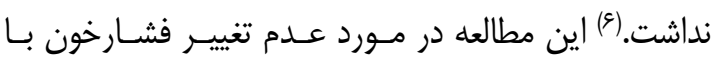

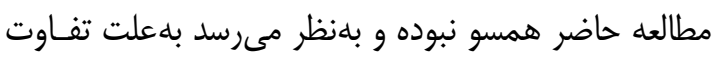

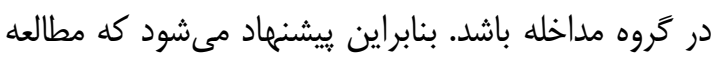
حاضر بر روى بيماران قلبى نيز انجام شود. انجام لمس بلهعنوان يك تحريك مناسب در بيمـاران

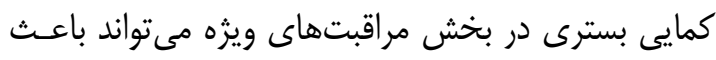

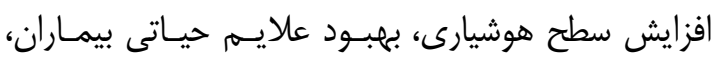
كاهش مدت زمـان بسـترى در ايـن بخـش و در نتيجـهـ

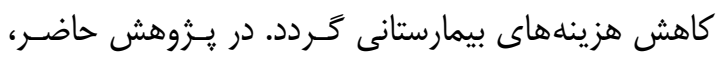

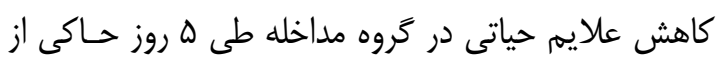

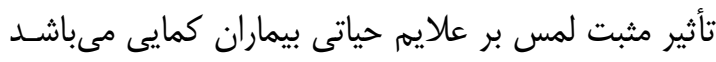

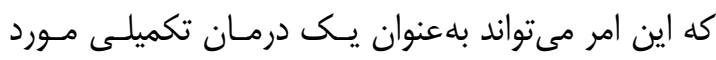

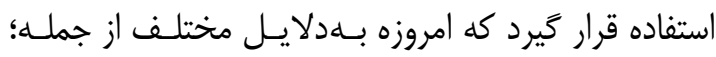

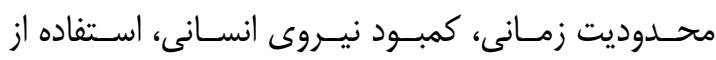

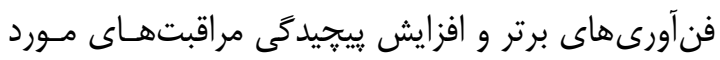

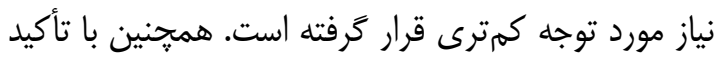

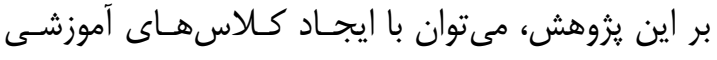

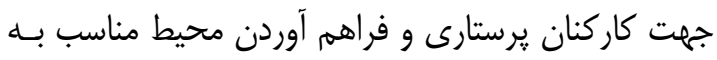

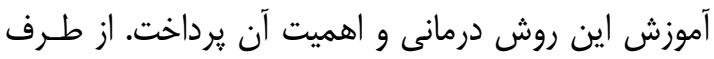

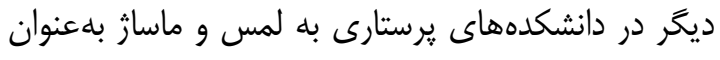

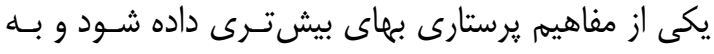

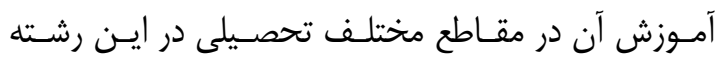

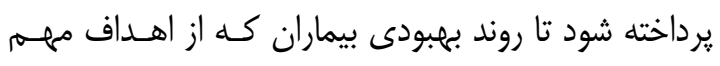

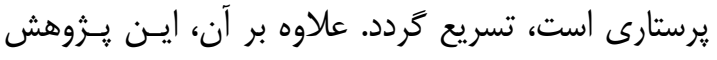


1. Yousefi H, Naderi M, Daryabeigi R. The effect of sensory stimulation provided by family on arterial blood oxygen saturation in critical care patients. Iran J Nurs Midwifery Res 2015; 20(1): 63-8.

2. Goudarzi F, Basampoor SH, ZakeriMoghadam M, Faghih-ZadehS, Rezaie F, Mohamad-Zadeh F. Changes in level of consciousness during auditory stimulation by familiar voice in comatose patients. J Iran Univ Med Sci 2010; 23(63): 43-50. [In Persian]

3. Fleischer S, Berg A, Behrens J, Kuss O, Becker R, Horbach A, et al. Does an additional structured information program during the intensive care unit stay reduce anxiety in ICU patients? a multicenter randomized controlled trial. BMC Anesthesiol 2014; 14: 48. doi: 10.1186/14712253-14-48.

4. Marino P. Marino's the ICU book. P moradi majd, $\mathrm{R}$ javadi, $\mathrm{F}$ behesht aeen. 1st ed. Tehran: Andisheh-rafi Publication; 2014. 566.

5. Dias Dde Sa, Resende MV, Diniz Gdo C. Patient stress in intensive care: comparison between a coronary care unit and a general postoperative unit. Rev Bras Ter Intensiva 2015; 27(1): 18-25. doi: 10. 5935/0103507X.20150005.

6. Zare Z, Shahsavari H, Moeini M. Effects of therapeutic touch on the vital signs of patients before coronary artery bypass graft surgery. Iran J Nurs Midwifery Res 2010; 15(1): 3742.

7. Adib-Hajbaghery M, Abasi A, RajabiBeheshtabad R. Whole body massage for reducing anxiety and stabilizing vital signs of patients in cardiac care unit. Med J Islam Repub Iran 2014; 28: 47.

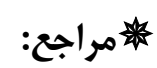

8. McGloin S, McLeod A .Advanced Practice in Critical Care. 1st ed. UK: Wiley-Blackell Publication; 2010. 228.

9. Vahedian-Azimi A, Ebadi A, Asghari Jafarabadi M, Saadat S, Ahmadi F. Effect of massage therapy on vital signs and GCS scores of ICU patients: a randomized controlled clinical trial. Trauma Mon 2014; 19(3): e17031. doi: 10.5812/traumamon. 17031.

10. Hefny AF, Idris K, Eid HO, Abu-Zidan FM. Factors affecting mortality of critical care trauma patients. Afr Health Sci 2013; 13(3): 731-5. doi: 10.4314/ahs.v13i3.30.

11. Abdi M. The effect of foot massage on the level of consciousness in comatose patients with brain injury hospitalized in ICU. Master's Thesis for critical Care nursing. Nursing and Midwifery College-Qazvin University of Medical science. 2014. [In Persian]

12. Fazel M, Fakhariyan A, Tabesh $\mathrm{H}$, Gharghani Z, Yeganeh Moghadam A, Mousavi Gh. Traumatic brain injury in Kashan during 2004-2005. Feyz 2009; 12(5): 21-6. [In Persian]

13. Aghakhani N, Azami M, Jasemi M, Khoshsima M, Eghtedar S, Rahbar N. Epidemiology of traumatic brain injury in Urmia, Iran. Iran Red Crescent Med J 2013; 15(2): 173-4. doi: 10.5812/ircmj.2090.

14. Abbasi M, Mohammadi E, Sheykh Rezayi A. Effect of a regular family visiting program as an affective, auditory, and tactile stimulation on the consciousness level of comatose patients with a head injury. Jpn J Nurs Sci 2009; 6(1): 21-6. doi: 10.1111/j. 1742-7924.2009.00117.x.

15. Patricia A. Potter P, Perry AG, stokert $P$, Hall A. Fundamentals of Nursing. 9th ed 
Mosby; 2017: 643-4

16. Souri Lakie A, Bolhasani1 M, Nobahar M, Fakhr Movahedi A, Mahmoudi H.The effect of touch on the arterial blood oxygen saturation in agitated patients undergoing mechanical ventilation. Iran J Crit Care Nurs 2012; 5(3): 125-32.

17. Memarian R. Application of nursing concepts and theories. 3rd ed. Tehran, Heydari Publication; 2014. 346. [In Persian]

18. Asad Zandi M. Concept and theories of nursing. 1st ed. Tehran, Heydari Publication; 2014. 409. [In Persian]

19. Nobahar M, Bolhasani M, FakhrMovahedi A, Ghorban R. Effects of touch on agitation in patients under mechanical ventilation. Koomesh 2014; 15(3): 325-33. [In Persian]

20. Fakhr-Movahedi A, Nobahar M, Bolhasani M. The effect of touch on the vital signs of agitated patients undergoing mechanical ventilation: an interventional study. J Urmia Nurs Midwifery Fac 2015; 12(10): 899-97. [In Persian]

21. Megha M, Harpreet S, Nayeem Z. Effect of frequency of multimodal coma stimulation on the consciousness levels of traumatic brain injury comatose patients. Brain Inj 2013; 27(5): 570-7. doi: 10.3109/02699052.2013. 767937.

22. Hasanzadeh F, Hoseini Azizi T, Esmaily $\mathrm{H}$, Ehsaee MR. The impact of familiar sensory stimulation on level of consciousness in patients with head injury in ICU. J North Khorasan Univ Med Sci 2012; 4(1): 121-3. [In Persian]

23. Hoseini Azizi T, Hasanzadeh F, Esmaily H, Ehsaee MR, Masoudynia M. The effect of family's supportive presence on the recovery of patients with brain injury in intensive care unit: a randomized clinical trial. J Urmia Nurs
Midwifery Fac 2014; 11(2): 977-87. [In Persian]

24. Schnakers C, Magee WL, Harris B. Sensory stimulation and music therapy programs for treating disorders of consciousness. Front Psychol 2016; 7: 297. doi: 10.3389/fpsyg.2016.00297.

25. Heidari Gorji MA, Araghiyansc F, Jafari H, Heidari Gorgi AM, Yazdani J. Effect of auditory stimulation on traumatic coma duration in intensive care unit of Medical Sciences University of Mazandaran, Iran. Saudi J Anaesth 2014; 8(1): 69-72. doi: 10. 4103/1658-354X.125940.

26. Zolfaghari M, Eybpoosh S, Hazrati M. Effects of therapeutic touch on anxiety, vital signs, and cardiac dysrhythmia in a sample of Iranian women undergoing cardiac catheterization. J Holist Nurs 2012; 30(4): 225-34. doi: 10.1177/0898010112453325.

27. Hussain Rezaee H, Jahani Y, Pakzad Moghaddam SH, Monfared Sh, Ebrahimi Hooshyar A. Effect of stimulation program on consciousness of traumatic brain injury patients: a comparison of FOUR and GCS scale. J Iran Society Anesthesiol Intensive Care 2015; 37(90): 98-104. [In Persian]

28. Haji Hoseini F, Avazeh A, Elahi N, Shariati A, Sori H. The effect of massage on comatos patients' vital signs, hospitalized in intensive care units. J Arak Univ Med Sci 2017; 9(3): 1-10. [In Persian]

29. Heydari Gorji MA, Araghian Mojarad F, Jafari H, Gholipour A, Yazdani Cherati J. Comparing the effects of familiar and unfamiliar voices as auditory sensory stimulation in level of consciousness among traumatic comatose patients in intensive care unit. J Mazandaran Univ Med Sci 2013; 23(97): 208-14. [In Persian] 